\title{
Thrombose des sinus veineux cérébraux compliquée par une thromboangéite oblitérante
}

\author{
Stefan Einsiedler MD, Georg Hödl MD, Raffi Topakian MD
}

Citation : CMAJ 2021 March 1;193:E311. doi : 10.1503/cmaj.201166-f

Voir la version anglaise de l'article ici : www.cmaj.ca/lookup/doi/10.1503/cmaj.201166

$\mathbf{U}$

n homme de 51 ans est venu consulter à notre hôpital pour des maux de tête, des nausées et une vision floue de novo persistant depuis 4 jours. Le patient, un fumeur, avait pour seul antécédent médical une thromboangéite oblitérante (aussi appelée «maladie de Buerger ») ayant nécessité une amputation tibiale gauche. Sa médication se résumait à $100 \mathrm{mg} / \mathrm{j}$ d'acide acétylsalicylique. À l'examen, le patient présentait un œdème papillaire bilatéral, mais aucun déficit neurologique focal. Une phlébographie par tomodensitométrie a révélé une thrombose des sinus veineux cérébraux (TSVC) (figure 1). Outre une thromboangéite oblitérante, les analyses sanguines n'ont mis en évidence aucune maladie infectieuse, auto-immune, néoplasique ou thrombophilique. Les symptômes du patient, à l'exception de l'œdème papillaire, se sont résorbés dans la semaine suivant l'amorce d'une anticoagulothérapie à la daltéparine. D'autres examens de neuroimagerie réalisés avant le passage à une anticoagulothérapie orale ont permis d'exclure la possibilité d'une hémorragie veineuse secondaire au traitement, malgré la persistance d'une importante TSVC (figure 2).

Les TSVC représentent environ $1 \%$ des accidents vasculaires cérébraux, touchant 5 personnes sur 1 million chaque année ${ }^{1}$. Quatre-vingts pour cent des cas surviennent chez les personnes de moins de 50 ans; les facteurs de risque concernent tout le spectre des thrombophilies acquises et génétiques ${ }^{1}$. Les patients présentent généralement des maux de tête et des troubles visuels qui vont en s'aggravant, mais certains développent des déficits neurologiques focaux ou présentent des convulsions ou une altération de l'état de conscience. La présence concomitante de maux de tête et d'un œdème papillaire, en particulier chez une personne que l'on sait atteinte de thrombophilie, devrait renforcer les soupçons de TSVC. Ce trouble peut toutefois passer inaperçu si des examens de neuroimagerie sont demandés, mais qu'ils ne sont pas accompagnés d'une phlébographie; ce peut être le cas, par exemple, chez les patients qui ne présentent que des maux de tête ayant commencé de façon insidieuse et n'étant accompagnés d'aucun autre signe ou symptôme. En outre, la variabilité anatomique des sinus veineux fait en sorte que la sensibilité de la tomodensitométrie (TDM) n'est pas suffisamment élevée pour permettre le diagnostic de TSVC; en effet, seulement $30 \%$ des cas de TSVC donnent des résultats anormaux à la TDM

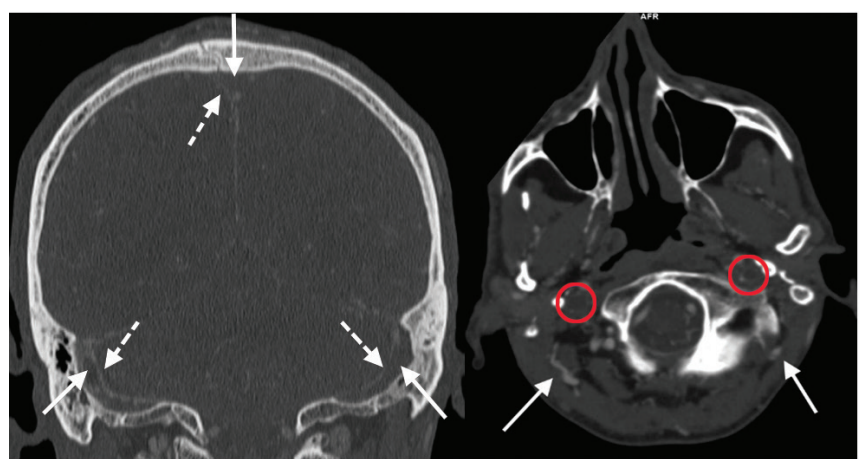

Figure 1 : Phlébographie par tomodensitométrie réalisée chez un homme de 51 ans à la consultation initiale. L'image de gauche montre une occlusion (flèches pleines) du sinus sagittal supérieur et des 2 sinus sigmoïdes, ainsi qu'une légère augmentation du contraste aux extrémités (flèches pointillées). L'image de droite montre une occlusion des 2 veines jugulaires internes (cercles) et un drainage veineux par les veines occipitales (flèches).

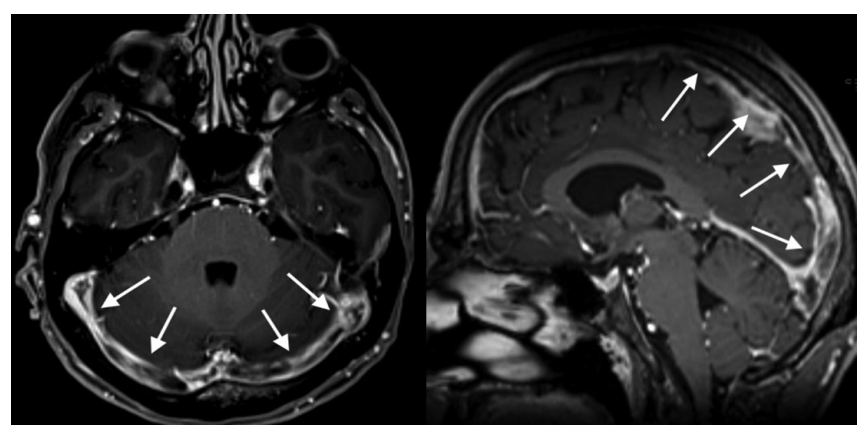

Figure 2 : Phlébographie par résonance magnétique réalisée 15 jours après la consultation initiale. On y observe une thrombose persistante des veines en pont, des 2 veines jugulaires internes, du sinus sagittal supérieur et des sinus transverses (flèches).

seule $^{1}$. Les bilans systémiques repèrent des causes prothrombotiques chez les deux tiers des patients ${ }^{1}$. La plupart des patients ont un excellent pronostic après l'amorce rapide d'une anticoagulothérapie, qui freine la progression de la TSVC et améliore le drainage veineux ${ }^{1}$.

La thromboangéite oblitérante est une maladie inflammatoire segmentaire rare touchant les artères et les veines de petit 
et de moyen calibres des extrémités, mais parfois aussi les vaisseaux cérébraux ${ }^{2,3}$. Elle est caractérisée par la formation de thrombus occlusifs inflammatoires contenant de nombreuses cellules; l'atteinte de la paroi des vaisseaux est relativement modeste. Les patients sont souvent des hommes d'âge moyen qui fument et qui présentent une ischémie distale aux membres ainsi que des ulcères aux doigts. La coexistence d'une thromboangéite oblitérante et d'une TSVC est extrêmement rare.

\section{Références}

1. Saposnik G, Barinagarrementeria F, Brown RD Jr, et al. Diagnosis and management of cerebral venous thrombosis: a statement for healthcare professionals from the American Heart Association/American Stroke Association. Stroke 2011;42:1158-92.

2. Bischof F, Kuntz R, Melms A, et al. Cerebral vein thrombosis in a case with thromboangiitis obliterans. Cerebrovasc Dis 1999;9:295-7.

3. Rivera-Chavarría IJ, Brenes-Gutiérrez JD. Thromboangiitis obliterans (Buerger's disease). Ann Med Surg (Lond) 2016;7:79-82.
Intérêts concurrents : Aucun déclaré.

Cet article a été révisé par des pairs.

Les auteurs ont obtenu le consentement du patient.

Affiliations : Service de neurologie (Einsiedler, Topakian) et Institut de radiologie (Hödl), Centre hospitalier universitaire Wels-Grieskirchen, Wels, Aut.

Propriété intellectuelle du contenu : Il s'agit d'un article en libre accès distribué conformément aux modalités de la licence Creative Commons Attribution (CC BY-NC-ND 4.0), qui permet l'utilisation, la diffusion et la reproduction de tout médium à la condition que la publication originale soit adéquatement citée, que l'utilisation se fasse à des fins non commerciales (c.-à-d., recherche ou éducation) et qu'aucune modification ni adaptation n'y soit apportée. Voir : https://creativecommons.org/licenses/by-nc-nd/4.0/deed.fr.

Correspondance : Raffi Topakian, raffi.topakian@hotmail.com 